\title{
Solution of a Class of Minimal Surface Problem with Obstacle
}

\author{
Kefei Liu (Corresponding author) \\ Department of Mathematics, Beihang University \\ Beijing 100083, China
}

Tel: 86-10-8231-7238Ｅ-mail: liukefei@yeah.net

Shangwei Zhao

\begin{abstract}
Key Laboratory of Mathematics Mechanization, Institute of Systems Science
AMSS, Chinese Academy of Sciences

Beijing 100080, China
\end{abstract}

Tel: 86-10-8268-3600 E-mail: zsw2011@126.com

\author{
Meizhu Liu \\ Department of Computer Information Science and Engineering \\ University of Florida \\ Gainesville, FL 32611, USA
}

Tel: 1-352-392-5770 E-mail: mliu@ cise.ufl.edu

\begin{abstract}
Plateau's problem is to determine the surface with minimal area that lies above an obstacle with given boundary conditions. In this paper, a special example of this class of the problem is given and solved with the linear finite element method. First, we triangulate the domain of definition, and transform the linear finite element approximation into a constrained nonlinear optimization problem. Then we introduce a simple and efficient method, named sequential quadratic programming, for solving the constrained nonlinear optimization problem. The sequential quadratic programming is implemented by the fmincon function in the optimization toolbox of MATLAB. Also, we discuss the relations between the number of grids and the computing time as well as the precision of the result.
\end{abstract}

Keywords: Minimal surface problem with obstacle, Finite element approximation, Constrained nonlinear optimization, Sequential quadratic programming

\section{Introduction}

Plateau's problem is to determine the surface of minimal area with a given closed curve in $R^{3}$ as boundary (Elizabeth, etc., 2004, pp.39-40). Suppose that the surface can be represented in nonparametric form $z: R^{2} \rightarrow$ $R$, and the requirement is $z \geq z_{L}$ for some obstacle $z_{L}$. The solution of this obstacle problem minimizes the function $f: K \rightarrow R$

$$
f(z)=\int_{D} \sqrt{1+\|\nabla z(x)\|^{2}} d x
$$

over the convex set

$$
K=\left\{z \in H^{1}(D) \mid z(x)=z_{D}(x) \text { for } x \in \partial D, z(x) \geq z_{L}(x) \text { for } x \in D\right\}
$$


where $\|\bullet\|$ represents the Euclidean norm, $H^{1}(D)$ is the space of functions with gradients in $L^{2}(D)$. The function $z_{D}: \partial D \rightarrow R$ defines the boundary data, and $z_{L}: D \rightarrow R$ is the obstacle. We assume that $z_{L} \leq z_{D}$ on the boundary $\partial D$.

The linear finite element approximation to the minimal surface with obstacle, defined by (1) and (2), can be obtained by triangulating $\mathrm{D}$ and minimizing $\mathrm{f}$ over the space of piecewise linear functions. The linear finite element approximation for the minimal surface with obstacle is analyzed, the existence and uniqueness of the solution for the discrete problem are shown, and the error estimate of the finite element approximation is obtained (Shen, etc., 1992, pp. 42-51).

In this paper, we intend to solve an example of the problem with the linear finite element approximation. In the example, we set $D=[0,1] \times[0,1]$, and use the boundary data

$$
z_{D}(x, y)= \begin{cases}1-(2 x-1)^{2} & y=0,1 \\ 0 & \text { otherwise }\end{cases}
$$

and the obstacle

$$
z_{L}(x, y)= \begin{cases}1 & \text { if } \sqrt{\left(x-x_{0}\right)^{2}+\left(y-y_{0}\right)^{2}} \leq 0.25 \\ 0 & \text { otherwise }\end{cases}
$$

where $\left(x_{0}, y_{0}\right)$ is the center of the projection circle on X-Y plane of the obstacle. The question is: when $\left(x_{0}, y_{0}\right)=(0.5,0.5)$, what is the minimal area? And when $\left(x_{0}, y_{0}\right)$ can move freely within $\mathrm{D}$, what is the maximum minimal area?

\section{Mathematic Model}

To obtain the linear finite element approximation to the minimal surface, we use a triangulation with, respectively, $n_{x}$ and $n_{v}$ internal grid points in the coordinate directions, as shown in Figure 1, where $(\mathrm{m}, \mathrm{n})$ represents the index of the coordinate data.

We set $n_{x}=n_{v}=n$ for simplicity, and let $z_{i j}$ denote $z\left(x_{i j}, y_{i j}\right)$. Thus the surface area is approximately $2 n^{2}$ small triangle area, and the linear finite element approximation of the problem (1)(3)(4) may be transformed into the following constrained nonlinear optimization problem

$$
\min _{z_{i j}} S=\sum_{k=1}^{2 n^{2}} S_{k}\left(\left\{z_{i j}, i, j=1, \cdots, n+1\right\}\right)
$$

subject to

$$
\left\{\begin{array}{l}
z_{i 1}=0 \\
z_{1 i}=1-\left(2 x_{1 i}-1\right)^{2} \quad i=1,2, \cdots, n+1 \\
z_{i, n+1}=0 \\
z_{n+1, i}=1-\left(2 x_{n+1, i}-1\right)^{2}
\end{array}\right.
$$

and

$$
z_{i, j} \geq \begin{cases}1 & \text { if } \sqrt{\left(x_{i j}-x_{0}\right)^{2}+\left(y_{i j}-y_{0}\right)^{2}} \leq 0.25 \quad i, j=1,2, \cdots, n+1 \\ 0 & \text { otherwise }\end{cases}
$$

The minimum of this class of constrained nonlinear multivariable function can be solved by the fmincon function of MATLAB.

\section{Solving Algorithm}

The fmincon function uses the sequential quadratic programming (SQP) method to solve Medium-Scale Optimization problem of the following form

$$
\begin{array}{ll}
\min _{x} & f(x) \\
\text { s.t. } & g_{u}(x) \leq 0 \quad(u=1,2, \cdots, p) \\
& h_{v}(x)=0 \quad(v=1,2, \cdots, m)
\end{array}
$$


The recursive procedure of the SQP method is as follows (Li, 2006, pp. 117-119; Zhang, etc., 2007, pp. 117-122):

Give the initial point $x_{0}$, the initial Hessian matrix $H_{0}=I$ (identity matrix), and set $\mathrm{k}=0$.

(1) A QP problem of the following form is solved to get $d_{k}$.

$$
\begin{array}{lll}
\min _{d \in R^{n}} & \nabla f\left(x_{k}\right)^{T} d+\frac{1}{2} d^{T} H_{k} d \\
\text { s.t. } & g_{u}\left(x_{k}\right)+\nabla g_{u}\left(x_{k}\right)^{T} d \leq 0 \quad(u=1,2, \cdots, p) \\
& h_{v}\left(x_{k}\right)+\nabla h_{v}\left(x_{k}\right)^{T} d=0 \quad(v=1,2, \cdots, m)
\end{array}
$$

(2) Use linear search to form a new iterate

$$
x_{k+1}=x_{k}+\alpha_{k} d_{k}
$$

where the step length $\alpha_{k}$ is determined according to certain rules.

(3) Update the Hessian matrix using the BFGS formula of the Quasi-Newton method.

$$
H_{k+1}=H_{k}+\frac{q_{k} q_{k}^{T}}{q_{k}^{T} s_{k}}-\frac{H_{k} H_{k}^{T}}{s_{K}^{T} H_{k} s_{k}}
$$

where

$$
\begin{gathered}
s_{k}=x_{k+1}-x_{k} \\
q_{k}=\nabla f\left(x_{k+1}\right)+\sum_{i=1}^{m} \lambda_{i} \nabla g_{i}\left(x_{k+1}\right)-\left[\nabla f\left(x_{k}\right)+\sum_{i=1}^{m} \lambda_{i} \nabla g_{i}\left(x_{k}\right)\right]
\end{gathered}
$$

and $\lambda_{i}(i=1, \cdots, m)$ is an estimate of the Lagrange multipliers.

\section{Results and Analysis}

4.1 The minimal area when $\left(x_{0}, y_{0}\right)=(0.5,0.5)$

Write MATLAB program using the fmincon function (choose Medium-Scale Algorithm), and run it to get the results. The minimal surface is shown in Figure 2. The minimal surface area is $\min S=2.4075$. It can be seen from Figure $2 \mathrm{~b}$ ) that the surface is sunken in the y direction.

The minimal surface area depends on the number of the grid points. Figure 3 shows the relationship between the minimal area and the number of grid points.

As can be seen from Figure 3, with the increase of the number of grid points, the minimal area is increasing on the whole. This is because the area of a triangular element unit is smaller than that of the curved surface, and the approximate error is reduced with more grid points, as shown in Figure 4. And the volatility is weakening, i.e. it has the trend to a constant. This is in line with the theoretical result of (Shen, Shumin, 1992), which concludes that the finite element approximate solution converges to the true solution when the number of grid points tends to infinity.

In addition, with the increase of the number of grid points, the number of the variable is increasing, so the computation becomes slower. For example, it takes only 37.48s for 10 grid points to finish computation, an hour for 35 grid points, while about 4 hours for 40 grid points. The computed result also depends on the parameters of the fmincon function. In the experiment, TolFun(termination tolerance on the function value), TolCon(termination tolerance on the constraint violation) and TolX (termination tolerance on $\mathrm{x}$ ) are all set to be $10^{-5}$.

\subsection{The maximum minimal area when moves freely within $D$}

In this case, $x_{0}, y_{0}$ can be treated as extra parameters to be optimized. Due to symmetry of the boundary data, it only need to consider the case when $\left(x_{0}, y_{0}\right) \in[0,0.5] \times[0,0.5]$.

Write MATLAB program using the fmincon function (choose Medium-Scale Algorithm), and run it to get the results. As shown in Figure 5 (30 grid points), the maximum minimal area is obtained when $\left(x_{0}, y_{0}\right)=$ $(0.25,0.5)$. The corresponding maximum minimal area is $\operatorname{maxmin} S=2.5158$. 
From the above analysis, we have known that the minimal surface is sunken in the y direction. Obviously the surface with minimal area without obstacle is as Figure 6 shows. With the center of the plate obstacle approaching the y axis, the surface is bulging more, and the area of the surface will increase accordingly. So it is reasonable that the maximum minimal area is obtained when the plate obstacle is tangent to the $\mathrm{y}$ axis.

The maximum minimal area can also be obtained with $\left(x_{0}, y_{0}\right)=(0.75,0.5)$ for symmetry. Computed result that the minimal area is also 2.5158 confirms the conclusion. The corresponding surface (30 grid points) is shown in Figure 7.

\section{Conclusion}

This paper solves the minimal surface problem with obstacle with linear finite element approximation. We transform the minimal surface problem with obstacle into a constrained nonlinear optimization problem. Our method is based on the sequential quadratic programming method, and we use the fmincon function in the optimization toolbox of MATLAB. Based on the results, the relation between the number of grid points and precision of the results as well as computation time is discussed.

\section{References}

Elizabeth D. Dolan, Jorge I. More, \& Todd S. Munson. (2004). Benchmarking Optimization Software with COPS 3.0. [Online] Available: http://www.osti. gov/bridge (August 5, 2008).

Shen, Shumin, \& Deng, Qingping. Finite Element Method for the Minimal Surface Problem with Obstacle. Numerical Mathematics A Journal of Chinese Universities, 1992, No. 1:42-51.

Li, Yuanke. (2006). Engineering Optimal Design. Beijing: Tsinghua University Press, (Chapter 6).

Zhang, Kecun, \& Li, Huanqin. Engineering Optimal Method and Its Application. Xi,an: Xi'an Jiaotong University Press, (Chapter 3).

Van der Geer, J., Hanraads, J. A. J. \& Lupton R. A. (2000). The art of writing a scientific article. Journal of Scientific Communications, 163, 51-59.

Strunk, W., Jr. \& White, E. B. (1979). The elements of style. (3rd ed.). New York: Macmillan, (Chapter 4).

Mettam, G. R. \& Adams, L. B. (1994). How to prepare an electronic version of your article. In B. S. Jones, \&

R. Z. Smith (Eds.), Introduction to the electronic age (pp. 281-304). New York: E-Publishing Inc.

Smith, Joe, (1999), One of Volvo's core values. [Online] Available: http://www.volvo.com/environment/index.htm (July 7, 1999).

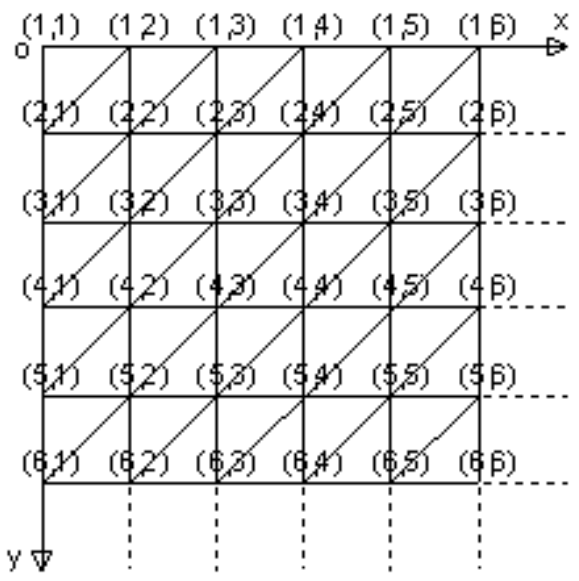

Figure 1. Triangulation of the region $D=[0,1][0,1]$ 


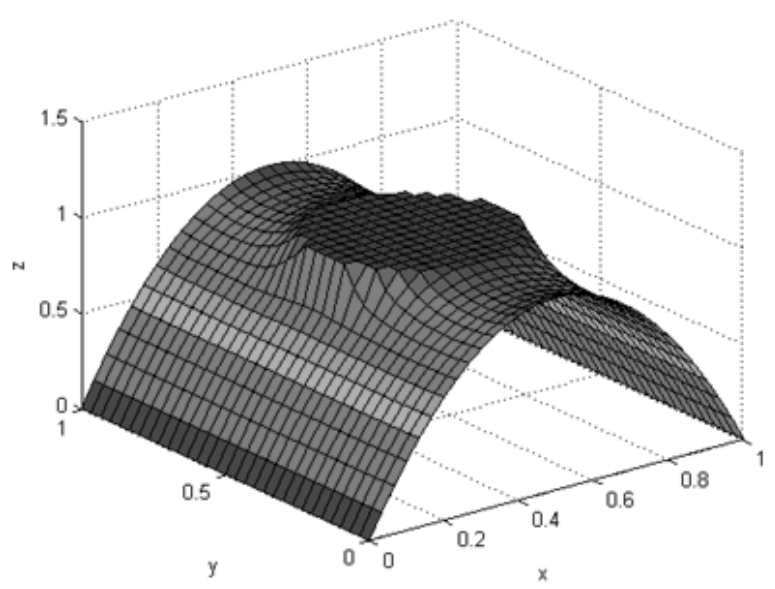

a) Three- dimensional graph

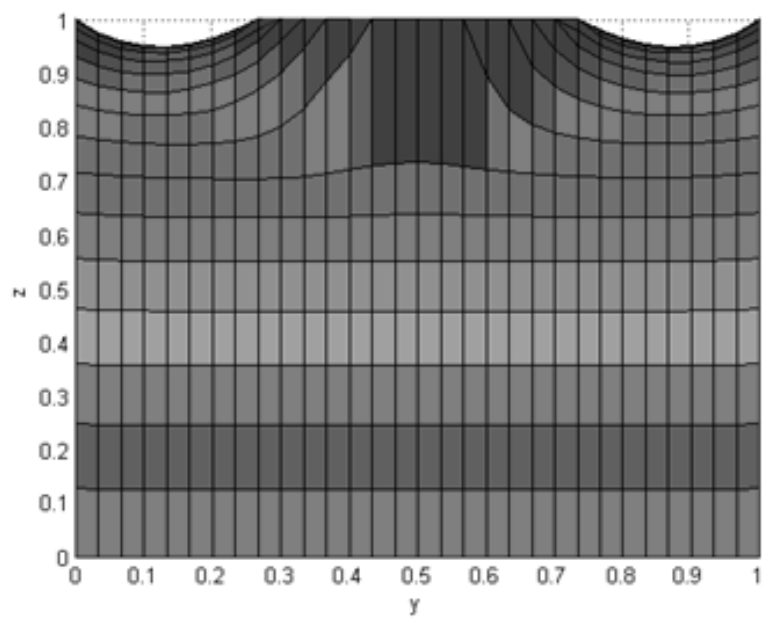

b) View from $x$ direction

Figure 2. Minimal surface with obstacle (30 grid points)

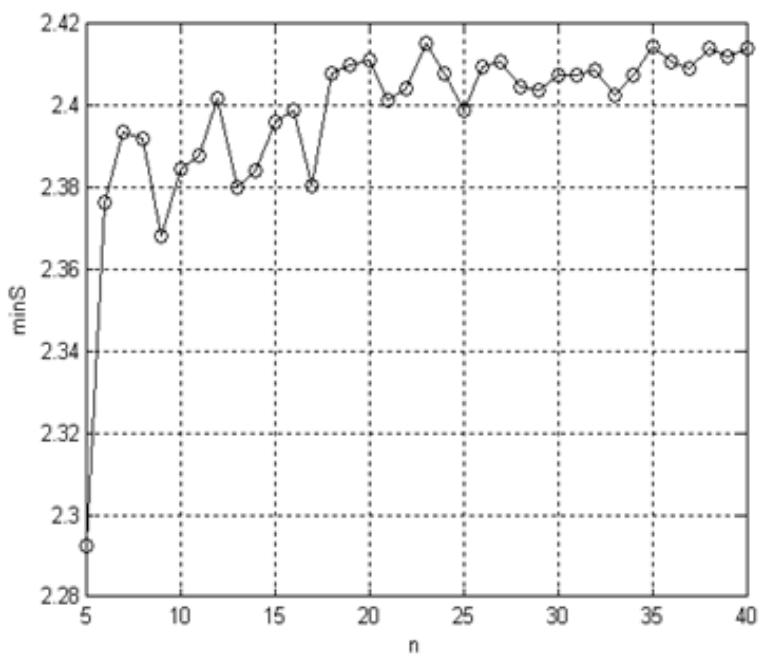

Figure 3. The relationship between the minimal area and the number of grid points 


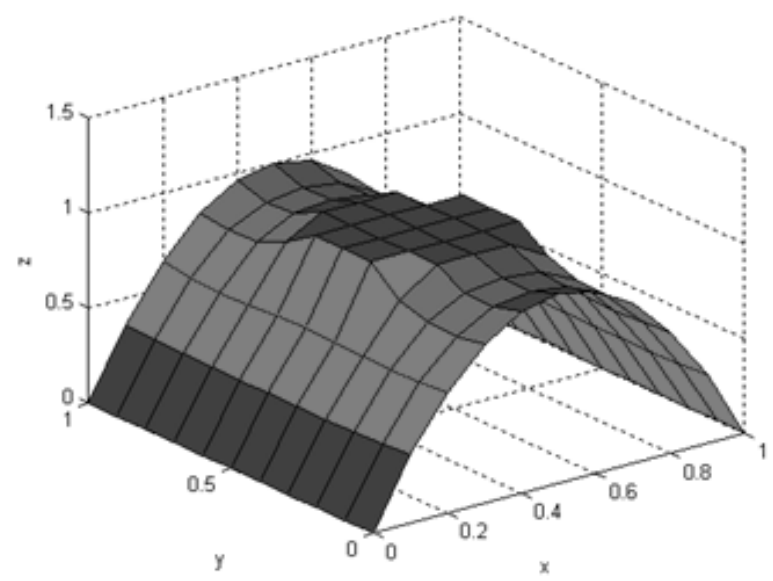

a) 10 grid points

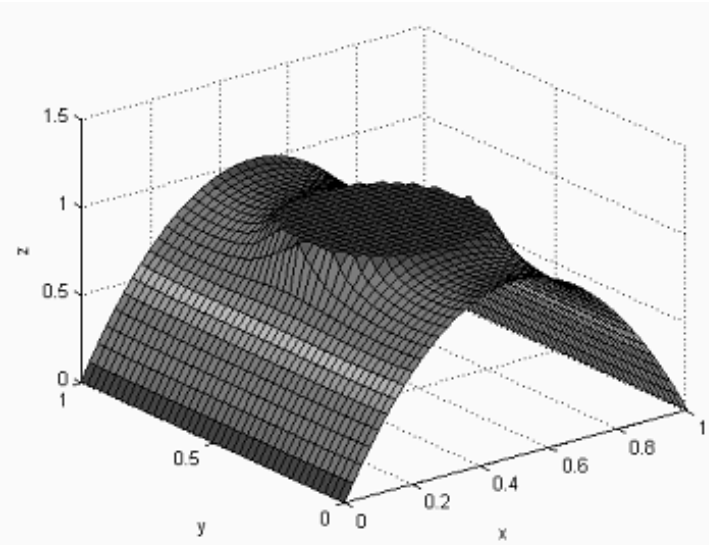

b) 40 grid points

Figure 4. Comparison of the minimal surface with 10 grid points and 40 grid points

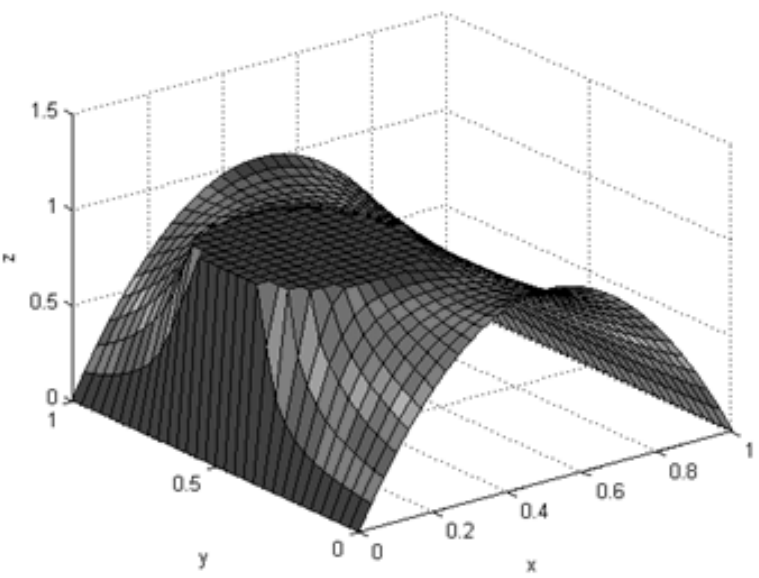

Figure 5. Surface with maximum minimal area 


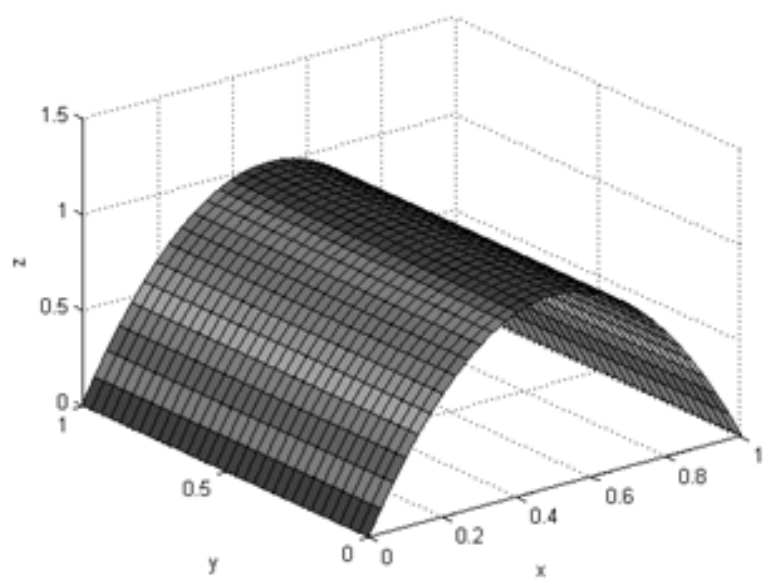

Figure 6. Minimal surface without obstacle

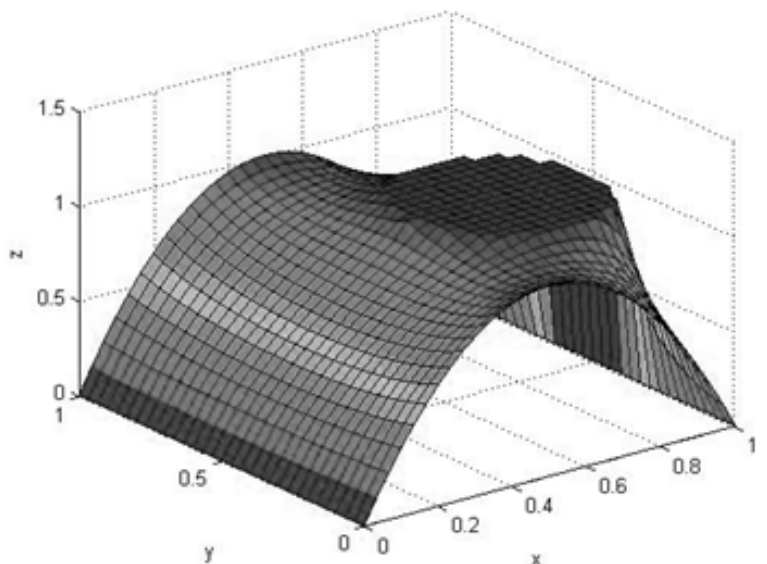

Figure 7. Surface with maximum minimal area 